\title{
Digestion des protéines de pois dans la caillette et l'intestin grêle du veau préruminant : résultats préliminaires
}

\author{
I Nunes do Prado', R Toullec1, P Guilloteau1', J Guéguen² \\ avec la collaboration technique de S Boussion et de M Formal \\ 1 Laboratoire du Jeune Ruminant, INRA, 65, rue de Saint-Brieuc, 35042 Rennes Cedex; \\ 2 Laboratoire de Biochimie et Technologie des Proteines, INRA, \\ BP 527, 44026 Nantes Cedex 03, France
}

Summary - In preruminant calves given a milk-substitute diet containing raw pea flour, the amounts of immunoreactive legumin leaving the abomasum and the ileum were found to be equivalent to about 24 and 3\% of intake, respectively. This long-term presence along the digestive tract could favor its allergenic effects.

Introduction - L'ingestion de pois cru par le veau préruminant provoque la formation d'anticorps dirigés contre les protéines alimentaires et une augmentation de la perméabilité intestinale aux macromolécules, qui serait le signe d'une altération de la paroi, probablement d'origine allergique (Nunes do Prado et al, 1989). La présence de protéines alimentaires peu ou pas digérées jusqu'à la fin de l'iléon est considérée comme favorisant les réactions de nature allergique observées chez certains veaux recevant du soja (Sissons et Thurston, 1984). Le but de ce travail est d'estimer la part de la légumine du pois parvenant sous forme immunoréactive (LI) à l'entrée et à la fin de l'intestin grêle.

Matériel et Méthodes - Des veaux préruminants sont munis soit d'un cathéter abomasal et d'une canule duodénale réentrante ( 3 veaux D), soit d'une canule réentrante à la fin de l'iléon (1 veau I). Deux aliments d'allaitement : $T$ et $P$, $19 \%$ de lipides et $25 \%$ de matières azotées (MA) par rapport à la matière sèche (MS) sont utilisés. La poudre de lait écrémé apporte la totalité des MA dans l'aliment $\mathrm{T}$; dans l'aliment $\mathrm{P}$, elle est remplacée en partie (32\%) par de la fa- rine de pois cru dépelliculé ( $27 \%$ de MAMS, dont $26 \%$ de $\mathrm{LI}$ et $10 \mathrm{U}$ de trypsine inhibée/mg de MA). Les veaux reçoivent 2 repas/j (a 8 h 30 et $16 \mathrm{~h} \mathrm{30}$ ) apportant chacun $29 \mathrm{~g}$ de $\mathrm{MS} / \mathrm{kg}$ de poids vifo,75. Chez les veaux $D$, l'aliment $P$ est infusé dans la caillette la veille au soir et le matin du jour des mesures. Les digesta sont recueillis en totalité dans un récipient entouré de glace fondante qui est relevé 15, 30, 60, 120, $180,240,300,360$ ef $420 \mathrm{~min}$ après le début de l'infusion. Une aliquote $(10 \%$ est transférée a $-18^{\circ} \mathrm{C}$ et lyophilisée. Le reste des digesta est infusé dans la partie distale de la canule, sauf en attendant la constitution de la première aliquote de digesta où l'on introduit du lait de remplacement. Au bout de $15 \mathrm{~min}$, puis a l'issue de chaque heure, quelques grammes de digesta sont recueillis séparément, immédiatement dilués dans 5 fois leur poids de tampon phosphate $\left(4^{\circ} \mathrm{C}, \mathrm{pH} 7,5\right)$ et mis a $-18^{\circ} \mathrm{C}$. Chez le veau I, l'aliment $P$ est substitué à l'aliment $T$ 6 j avant le début des prélèvements. Les digesta sont collectés et échantillonnés de 0 a $10 \mathrm{~h}$ après le repas du matin, pendant 3 j consécutifs, dans les mêmes conditions que pour les veaux $D$, sauf que les récipients sont relevés toutes les heures, que les prélèvements extemporanés ont lieu au milieu de chaque heure et que les digesta ne sont pas réintroduits. La LI est dosée par ELISA (à l'aide d'anticorps préparés contre la protéine native) dans le pois et les 
Tableau I. Teneur en quelques AA caractéristiques (\% de la somme des AA dosés) des protéines des aliments et des digesta.

\begin{tabular}{|c|c|c|c|c|c|c|}
\hline \multirow[t]{2}{*}{$A A$} & \multirow[t]{2}{*}{ Aliment $T$} & \multirow[t]{2}{*}{ Pois } & \multirow[t]{2}{*}{ Aliment $P$} & \multicolumn{3}{|c|}{ Digesta } \\
\hline & & & & duodénaux ${ }^{a}$ & iléaux a & iléaux b \\
\hline $\begin{array}{l}\text { Thréonine } \\
\text { Proline } \\
\text { Glycine } \\
\text { Arginine }\end{array}$ & $\begin{array}{l}4,83 \\
9,65 \\
1,82 \\
3,30\end{array}$ & $\begin{array}{l}3,74 \\
4,43 \\
4,46 \\
8,96\end{array}$ & $\begin{array}{l}4,41 \\
7,58 \\
2,73 \\
5,08\end{array}$ & $\begin{array}{l}4,19 \\
7,60 \\
2,64 \\
4,46\end{array}$ & $\begin{array}{l}7,82 \\
4,41 \\
4,63 \\
4,12\end{array}$ & $\begin{array}{l}8,96 \\
5,80 \\
5,55 \\
3,95\end{array}$ \\
\hline
\end{tabular}

a Avec l'aliment P. b Avec l'aliment T.

échantillons de digesta prélevés extemporanément. La composition en acides aminés (AA) est déterminée sur le pois, le reste de l'aliment $P$, l'aliment $T$, les échantillons de digesta obtenus en mélangeant pour chaque veau les aliquotes lyophilisées et un échantillon représentatif des digesta prélevés préalablement chez le veau I alors qu'il recevait l'aliment T. Les profils d'AA sont comparés par la distance du $\chi^{2}$ (Guilloteau et al, 1983).

Résultats et Discussion - La légumine quitte la caillette en quantité importante sous forme immunoréactive jusque vers $5,5 \mathrm{~h}$ après le repas, mais très peu audelà, probablement à cause de la baisse du $\mathrm{pH}(2,8 \pm 0,4$ (moyenne \pm écart type de la moyenne) après $5 \mathrm{~h}$, au lieu de $6,0 \pm 0,1$ après $15 \mathrm{~min} ; P<0,05)$. La quantité cumulée de $\mathrm{LI}$ recueillie au bout de $7 \mathrm{~h}$ est de $24,3 \pm 3,7 \%$ de la quantité ingérée. La composition en AA des digesta duodénaux (tableau I) est pratiquement identique à celle de l'aliment $P$ (distance du $\chi^{2}=7$ ). Par conséquent, en $7 \mathrm{~h}$, les diverses protéines alimentaires quittent globalement la caillette dans des proportions très voisines (environ $72 \%$ pour les MA totales). La coagulation de l'aliment $P$ est donc altérée puisque les protéines sériques sont normalement évacuées plus vite que les caséines (Yvon et al, 1986). La quantité de LI arrivant à la fin de l'iléon est très faible pendant les 4 premières heures postprandiales mais est notable au cours des $6 \mathrm{~h}$ suivantes: la quantité cumulée atteint $0,01 \pm 0,01$ et $2,7 \pm 1,1 \%$ de la quantité ingérée après respectivement 4 et $10 \mathrm{~h}$. Le $\mathrm{pH}$, voisin de 8 jusqu'à $4 \mathrm{~h}$, diminue ensuite jusqu'à $7 \mathrm{~h}(5,6 \pm 0,2 ; P<0,05)$, puis varie peu. La composition en AA des digesta iléaux est très différente de celles des protéines totales de l'aliment $P$ et des digesta duodénaux (distance du $\chi^{2}=181$ et 171) ou des diverses protéines du pois et du lait. En revanche, elle est proche de celle d'un mélange théorique comprenant $80 \%$ de protéines iléales obtenues avec l'aliment $\mathrm{T}$ et $20 \%$ de protéines de pois (distance du $\chi^{2}=30$ ).

En conclusion, une partie de la légumine parvient sous forme peu ou pas modifiée jusqu'à la fin de l'iléon. Ce long contact avec la muqueuse intestinale pourrait favoriser son absorption et son action allergénique éventuelle (Nunes do Prado et al, 1989).

Guilloteau $P$, Sauvant D, Patureau-Mirand $P$ (1983) Ann Nutr Métab 27, 457-459

Nunes do Prado I, Toullec R, Lallès JP, Hingand L, Guéguen J (1989) Reprod Nutr Dev 29, 413-424

Yvon M, Pélissier JP, Guilloteau P, Toullec R (1986) Reprod Nutr Dév 26, 705-715

Sissons JW, Thurston SM (1984) Res Vet Sci $37,242-246$ 